\title{
Medición de la fuerza de prensión y de las pinzas de la mano en pacientes sanos
}

\author{
Measurement of the pressure force and hand clamps in healthy patients \\ Vázquez-Alonso MF, ${ }^{*}$ Díaz-López JJ, ${ }^{\ddagger}$ Lázaro-Huerta $M,{ }^{\ddagger}$ Guamán-González $M{ }^{\S}$ \\ Instituto Mexicano del Seguro Social.
}

RESUMEN. Introducción: La caracterización biomecánica de agarre supone la determinación de las diferentes variables cinemáticas y dinámicas que afectan sus distintas fases: transporte, formación del agarre y manipulación; su relación con las características antropométricas del sujeto y las propias del objeto. La fuerza de contacto en los dedos durante la realización de agarres bidigitales o multidigitales pero que involucran únicamente uso de las falanges distales (agarre tipo pinza) y el agarre de potencia cilíndrico son de los más empleados para las actividades diarias. La medición de la fuerza de presión (FPM) es un elemento esencial para seguimiento durante el crecimiento, lesiones, rehabilitación y ensayos terapéuticos. Material y métodos: Estudio descriptivo, prospectivo, transversal con 61 pacientes sin patología de miembro torácico, 30 hombres y 31 mujeres, con rango de edad de 20 a 59 años, se realizaron mediciones antropométricas y prueba de fuerza (kg/fuerza). Se analizaron las variables estadísticamente con «r» de Pearson y U de Mann-Whitney. Resultados: La fuerza de prensión de la mano y la pinza 1 de la mano dominante como la no dominante está correlacionada con el peso, talla, porcentaje de grasa corporal y circunferencia de la muñeca, la FPM y la pinza 1 tanto en la mano dominante como en la no do-
ABSTRACT. Introduction: The biomechanical characterization of grip involves the determination of the different kinematic and dynamic variables that affect its different phases: transport, grip formation and manipulation, as well as its relationship with the anthropometric characteristics of the subject and those of the object. The contact force in the fingers during the making of bidigital or multi-digital grips but involving only the use of distal phalanges (grip type clamp) and cylindrical power grip are among the most used for daily activities. Hand grip force measurement is an essential element for follow-up during growth, injury, rehabilitation and therapeutic trials. Material and methods: Descriptive, prospective, crosssectional study with 61 patients without upper extremity pathology, 30 male, 31 female, with age range of 20 to 59 years, anthropometric measurements and Force test (kg/ strength) were performed. The variables were statistically analyzed with Pearson's «r» and Mann-Whitney's U. Results: The hand grip and clamp 1 Force of the dominant hand as non-dominant is correlated with weight, size, body fat percentage and wrist circumference, HGF and clamp 1 in both the dominant and non-dominant hand is higher in men than in women, with $\mathrm{p}<0.05$. Conclusions: The average

\section{Nivel de evidencia: IV}

* Jefe de Servicio de Cirugía de Mano.

* Médico adscrito al Servicio de Cirugía de Mano.

${ }^{\S}$ Médico residente de cuarto año de Ortopedia.

Unidad Médica de Alta Especialidad en Traumatología y Ortopedia Lomas Verdes.

Correspondencia:

Dra. Ma. Francisca Vázquez-Alonso

Av. Lomas Verdes Núm. 52, Col. Santa Cruz Acatlán,

C.P. 53150 Naucalpan de Juárez,

Tel. 53710888, ext. 28664

E-mail: francisca.vazqueza@imss.gob.mx

Recibido: 26-11-2020. Aceptado:21-02-2021.

Citar como: Vázquez-Alonso MF, Díaz-López JJ, Lázaro-Huerta M, Guamán-González MO. Medición de la fuerza de prensión y de las pinzas de la mano en pacientes sanos. Acta Ortop Mex. 2021; 35(1): 56-60. https://dx.doi.org/10.35366/100932 
minante es mayor en los hombres que en las mujeres, con p $<0.05$. Conclusiones: La FPM promedio de la mano dominante es mayor que en la no dominante para hombres y mujeres, relacionado con el peso, talla, porcentaje de grasa corporal y circunferencia de la muñeca.

Palabras clave: Mano, prensión, fuerza, medición, sano.

\section{Introducción}

Durante el año 2011, las lesiones traumáticas de mano ocuparon el primer lugar como causa de incapacidades por accidentes de trabajo en el IMSS, representando 36\% de los accidentes de trabajo registrados: de estos, 61,557 (53.1\%) corresponden a heridas, 23,829 (20.5\%) a traumatismos superficiales, 156,810 (13.6\%) a fracturas y esguinces, y 3,951 (3.4\%) a amputaciones. Estas lesiones dejaron incapacidades parciales permanentes en 3,591 casos (3.1\%) del total de los accidentes de trabajo. Lo cual da idea de la elevada incidencia de estos padecimientos y sus repercusiones económicas y sociales. Las manos son una herramienta de gran utilidad para el hombre, quien las utiliza para realizar funciones mecánicas, sensitivas, de protección e incluso de comunicación, sus múltiples utilidades las hacen susceptibles de sufrir daños.

La mano humana es uno de los sistemas mecánicos más complejos y versátiles que existen. La caracterización biomecánica del agarre supone la determinación de las dife-
HGF of the dominant hand is higher than in the nondominant for men and women. This is related to weight, size, body fat percentage and wrist circumference.

Keywords: Hand, grasp, strength, measurement, healthy.

rentes variables cinemáticas y dinámicas que afectan a las diferentes fases del agarre: transporte, formación de agarre y manipulación; así como la relación con las características antropométricas del sujeto y las propias del objeto manipulado y la tarea de manipulación.

Existen trabajos ${ }^{2,3,4,5,6,7,8}$ que analizan las fuerzas de contacto en los dedos durante la realización de agarres digitales o multidigitales pero que involucran únicamente el agarre con las falanges distales (agarres tipo pinzas).

El agarre de potencia cilíndrico es uno de los más empleados durante la realización de las actividades de la vida diaria, se trata de un agarre con contacto de superficie elevada de la mano y con el que se pueden conseguir fuerzas elevadas. Dada su importancia, la caracterización biomecánica de este agarre tiene gran interés, ya que la funcionalidad de la mano humana radica en que dicho agarre pueda ser realizado. $^{9}$

Se ha descrito una relación entre la fuerza de prensión y varios parámetros funcionales, clínicos, sicológicos o sicosociales en diferentes poblaciones, sobre todo en per-
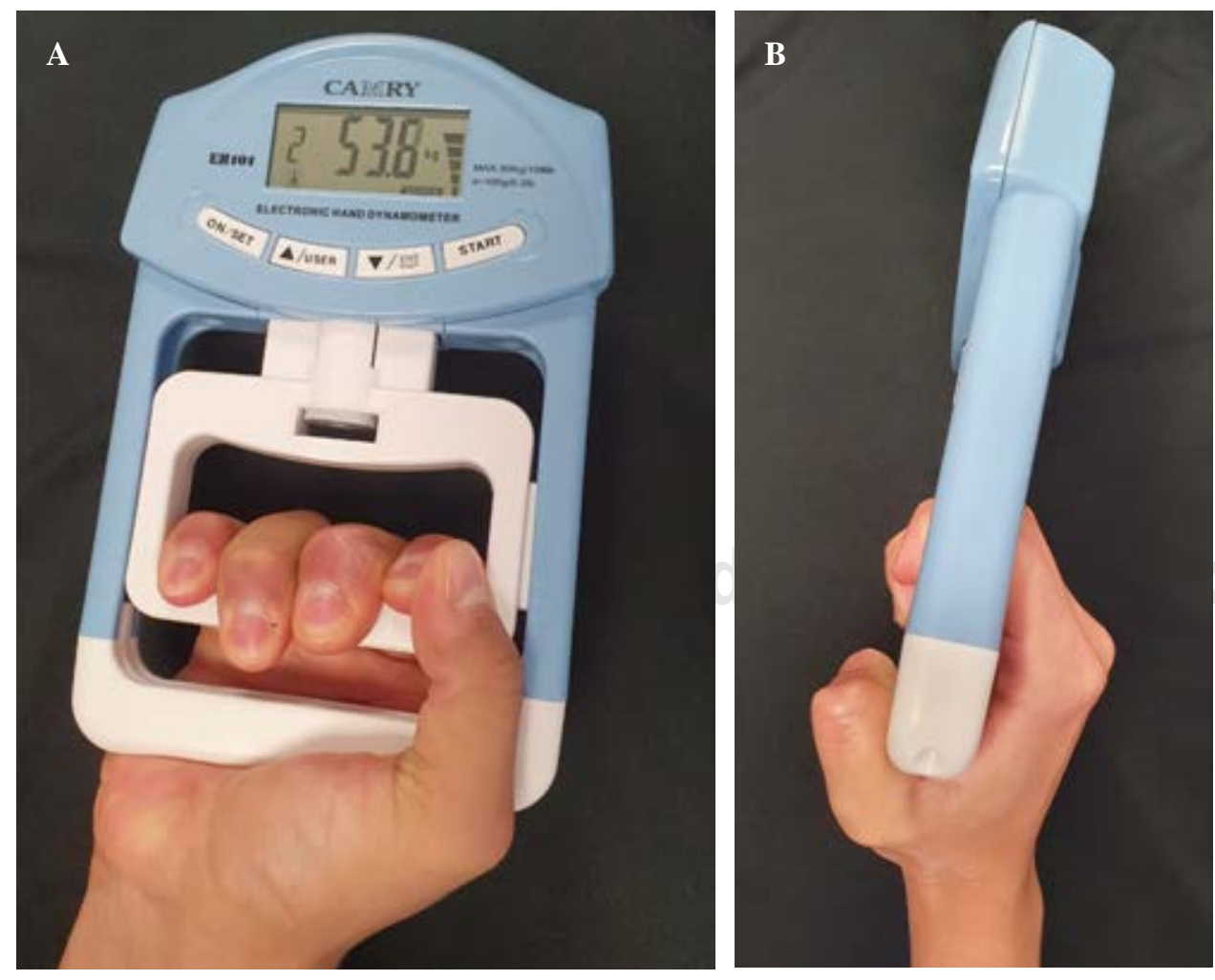

Figura 1:

Medición de la fuerza: A) De prensión. B) En puño. 


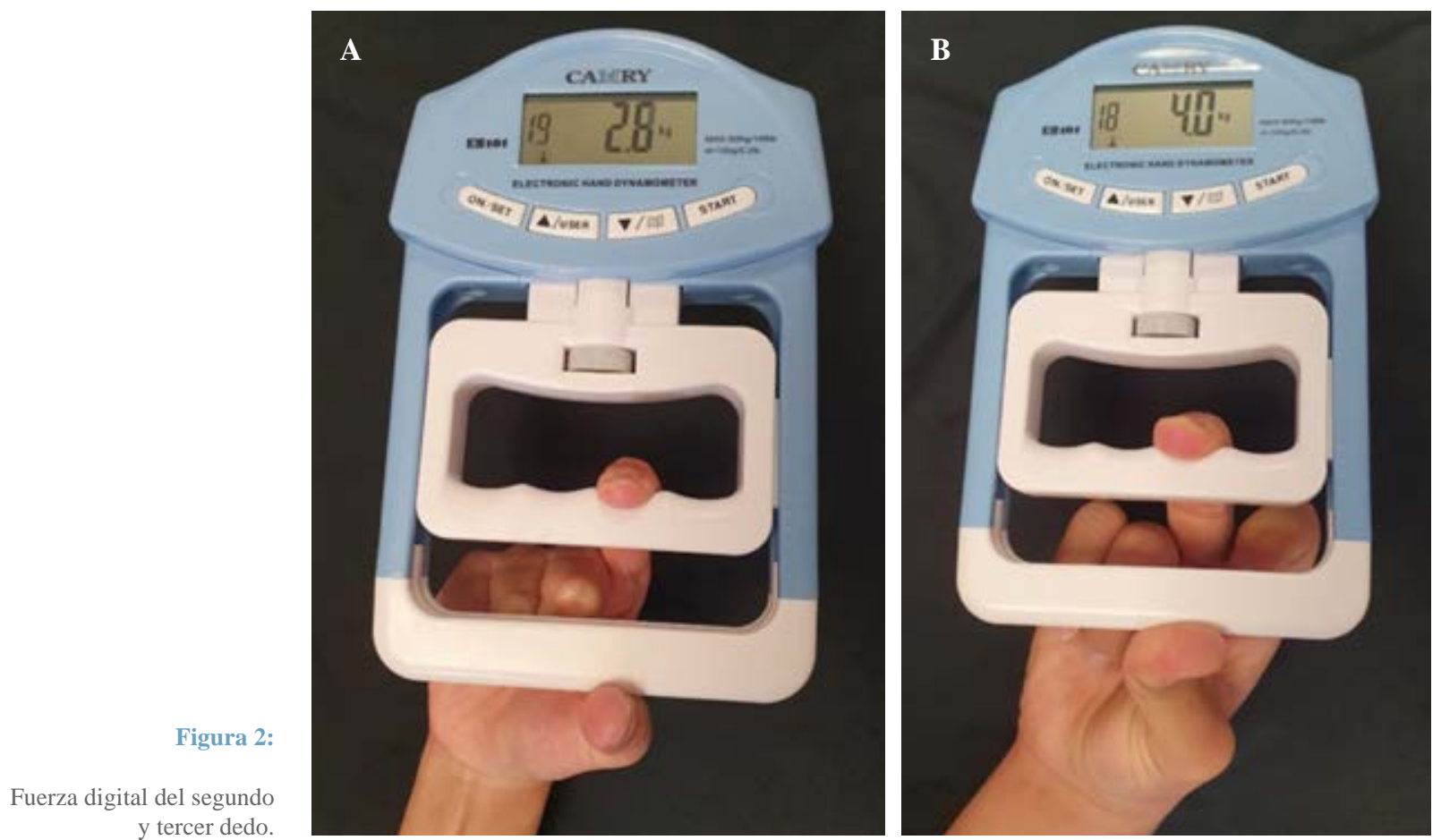

\begin{tabular}{|c|c|c|c|c|c|}
\hline & Prensión & $\begin{array}{c}\text { Pinza } \\
1\end{array}$ & $\begin{array}{c}\text { Pinza } \\
2\end{array}$ & $\begin{array}{c}\text { Pinza } \\
3\end{array}$ & $\begin{array}{c}\text { Pinza } \\
4\end{array}$ \\
\hline \multicolumn{6}{|c|}{ Fuerza dominante (kg/Fza) } \\
\hline Masculino & 51.72 & 9.92 & 8.44 & 6.76 & 5.40 \\
\hline Femenino & 31.68 & 8.00 & 7.56 & 6.32 & 4.96 \\
\hline \multicolumn{6}{|c|}{ Fuerza no dominante $(\mathrm{kg} / \mathrm{Fza})$} \\
\hline Masculino & 49.00 & 9.68 & 8.16 & 6.92 & 5.08 \\
\hline Femenino & 29.48 & 7.60 & 7.52 & 6.24 & 4.76 \\
\hline
\end{tabular}

sonas de edad avanzada. ${ }^{10}$ En relación a los tipos de presiones usadas, Kapandji las define de la siguiente forma: 1) prensiones palmares, tanto cilíndricas como esféricas, en las que intervienen los dedos y la palma de la mano, es una prensión de fuerza para tomar objetos pesados y voluminosos; 2) prensiones digitales, referida a la pinza digital subtérmino lateral, involucra pulgar e índice y permite tomar los objetos con precisión. ${ }^{11}$ La evaluación de la fuerza muscular forma parte de la valoración funcional de la mano junto con el análisis de la sensibilidad y la movilidad articular. La fuerza es definida como la capacidad de un músculo para ejercer el máximo de esfuerzo o resistir al poder de oposición. En la fuerza de presión manual, es el resultado de la flexión forzada de todas las articulaciones de los dedos. Existen diversas modalidades de valoración de la fuerza de la mano, habiéndose aplicando durante el transcurso del tiempo métodos de balanza en resorte, siste- mas de prensión, levantamiento de pesa, miómetro modificado y actualmente la dinamometría.

La medición de la fuerza de agarre máxima (MGS) es un elemento esencial para evaluar a la fuerza durante el crecimiento, envejecimiento, en las secuelas de lesiones traumáticas y en la rehabilitación. El dinamómetro es considerado un instrumento adecuado y confiable para la evaluación de la fuerza de prensión manual del paciente, aunque la fiabilidad de la evaluación puede verse afectada por género, peso y la postura corporal.

\section{Material y métodos}

Estudio realizado en el período comprendido de 01 de Abril al 5 de Noviembre 2017, prospectivo, descriptivo, transversal. Se incluyeron 61 pacientes sin patologías de la extremidad torácica conocida con un rango de edad entre 20 y 59 años de edad, ambos sexos, organizados en cuatro grupos etarios. Grupo 1, 20 a 29 años; grupo 2, de 30 a 39 años; grupo 3, de 40 a 49 años; y grupo 4, de 50 a 59 años. Se valoró la fuerza de prensión y de las pinzas de la mano mediante un dinamómetro hidráulico (Figuras 1 y 2); se tomó en cuenta índice de masa corporal, porcentaje de grasa corporal, circunferencia, flexión y extensión de la muñeca.

Se realizó estadística descriptiva para variables dicotómica y categóricas, se estableció la correlación con prueba de Pearson a variables cuantitativas y prueba de Spearman para variables categóricas y dicotómicas con un valor de correlación de $r=0.8$, además se realizó prueba de U de Mann Whitney con un valor significativo de $\mathrm{p}<0.5$. 


\section{Resultados}

Se estudiaron 30 hombres (49\%) y 31 mujeres (51\%). Dominancia 58 diestros (94\%) y tres zurdos (6\%); el grupo 1 estuvo compuesto por 26 personas (44\%); grupo 2, 23 personas (38\%); grupo 3, 10 personas (14\%); y grupo 4, 4 personas (4\%).

Se midieron variables de peso, talla, índice de masa corporal y porcentaje de grasa corporal, obteniendo un rango de talla de $1.40 \mathrm{~m}$ a $1.86 \mathrm{~m}$, con promedio de $1.65 \mathrm{~m}$, el índice de masa corporal con rango de 19.8 a $43.1 \mathrm{~kg} / \mathrm{m}^{2}$, con un promedio de $27.6 \mathrm{~kg} / \mathrm{m}^{2}$; por último se midió el porcentaje de grasa corporal con rangos de 14.14 a $52.6 \%$ con un promedio de $29.73 \%$.

En cuanto a la medición de la circunferencia de la muñeca, se obtuvo como promedio para la mano dominante 16.07 $\mathrm{cm}$ y para la no dominante $15.94 \mathrm{~cm}$, para la flexión de la muñeca dominante $73.14^{\circ} \mathrm{y}$ para la no dominante $72.34^{\circ}$, para la extensión de la dominante $76.44^{\circ}$ y la no dominante $76.5^{\circ}$.

Los resultados de la fuerza obtenida por género y grupos de edad se presentan en la Tablas 1 y 2 . El análisis por grupo de edad, género y fuerza del brazo dominante y no dominante se observa en la Tabla 3.

\section{Discusión}

El objetivo de este estudio fue establecer la fuerza de prensión y de las pinzas de la mano en paciente sin patologías de los miembros torácicos de manera manual mediante un dinamómetro a fin de establecer datos de referencia en la fuerza.

La relación que tiene la fuerza de prensión y la dominancia es de importancia, siendo la mayor fuerza en la mano dominante en $5-40 \%$ y puede estar o no relacionada con la actividad laboral. Schreuders y colaboradores ${ }^{12}$ mencionan respecto al género que la fuerza de prensión es mayor en

Tabla 2: Distribución de la fuerza por grupos de edad.

\begin{tabular}{lccccc}
$\begin{array}{l}\text { Grupo } \\
\text { de edad } \\
\text { (años) }\end{array}$ & Prensión & $\begin{array}{c}\text { Pinza } \\
1\end{array}$ & $\begin{array}{c}\text { Pinza } \\
2\end{array}$ & $\begin{array}{c}\text { Pinza } \\
3\end{array}$ & $\begin{array}{c}\text { Pinza } \\
4\end{array}$ \\
\hline \multicolumn{5}{c}{ Fuerza dominante (kg/Fza) } \\
\hline 20-29 & 43.00 & 9.09 & 7.82 & 6.27 & 4.91 \\
$30-39$ & 41.26 & 8.89 & 7.95 & 6.47 & 5.11 \\
$40-49$ & 43.29 & 9.29 & 9.29 & 8.14 & 6.43 \\
$50-59$ & 26.00 & 7.00 & 6.00 & 4.50 & 4.50 \\
\multicolumn{7}{c}{ Fuerza no dominante (kg/Fza) } \\
\hline $20-29$ & 41.95 & 8.95 & 7.73 & 6.27 & 4.64 \\
$30-39$ & 37.74 & 8.42 & 7.79 & 6.58 & 4.95 \\
$40-49$ & 38.86 & 8.86 & 8.71 & 8.00 & 6.00 \\
$50-59$ & 25.00 & 6.50 & 6.50 & 5.00 & 4.00 \\
\end{tabular}

Tabla 3: Distribución de grupo de edad y fuerza por pinzas.

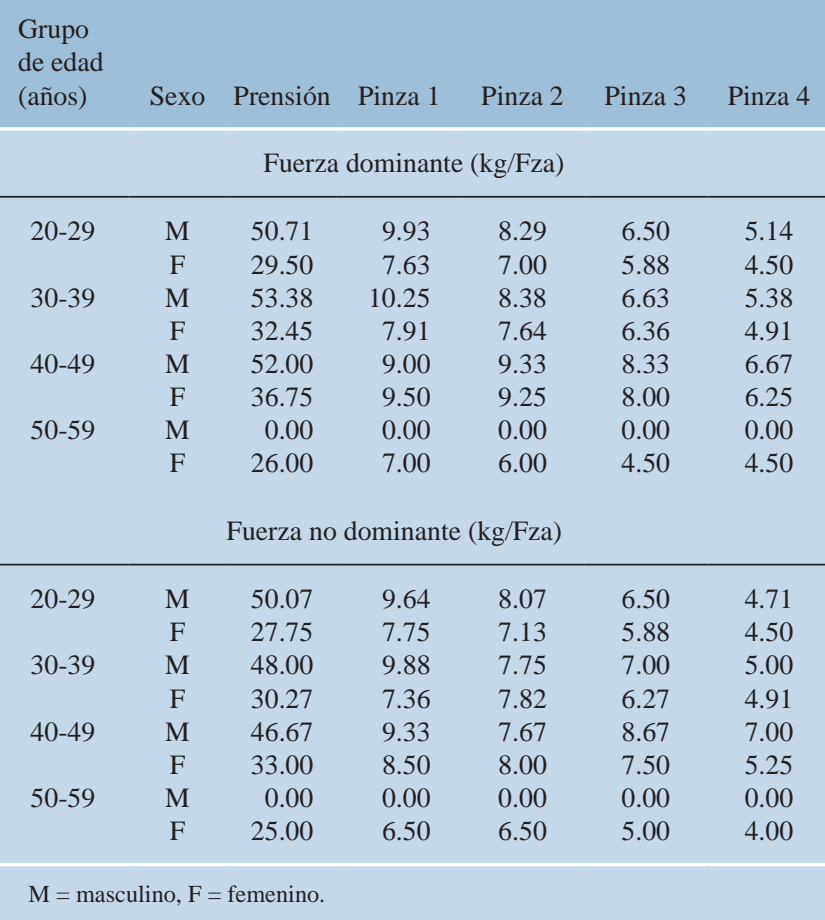

los hombres, hasta $60 \%$, Peters y su equipo ${ }^{13}$ dicen que la fuerza mayor es en personas entre la tercera y cuarta década de la vida, lo que concuerda con la edad productiva de la persona. ${ }^{14}$

En el presente trabajo, 50\% de los pacientes son del sexo masculino con predominancia diestra y relacionada con la tercera década de la vida laboral. La fuerza de presión en promedio es de $51.7 \mathrm{~kg} / \mathrm{Fza}$ en el sexo masculino en la mano dominante, en comparación con el sexo femenino que corresponde al $31.6 \mathrm{~kg} / \mathrm{Fza}$; en cuanto a la extremidad no dominante, en el los varones es $49.0 \mathrm{~kg} / \mathrm{Fza}$, y en las mujeres $29.48 \mathrm{~kg} / \mathrm{Fza}$, por lo que Betchol (1954), referido por Escalona, explica que existe $10 \%$ más de fuerza dominante que la no dominante.

De acuerdo con la antropometría, la población estudiada tiene un IMC en promedio de $27.6 \%$, lo cual nos habla de un sobrepeso; sin embargo, no hay correlación con la medición de la fuerza de presión. Por otro lado, la relación peso-talla/fuerza de prensión sí se encuentra relacionada con la extremidad dominante y a su vez con la pinza 1 en el grupo de edad de 30-39 y 40-49 años y presente en el sexo masculino. Esto tiene relación con el grupo de población económicamente productiva como lo explican Coronel y colaboradores. ${ }^{1,11}$

En la quinta y sexta décadas de la vida, se observa una disminución de la fuerza muscular e incremento del IMC, así como la disminución de la masa muscular e incremento del porcentaje de grasa, esto está relacionado con el proceso de envejecimiento y, por lo tanto, a la disminución de la actividad, tal como lo menciona Lenardt. ${ }^{15}$ 


\section{Conclusiones}

El análisis de la fuerza de prensión y de las pinzas de la mano en paciente sano es un marco de referencia para valorar la fuerza y las diferentes pinzas de la mano en la patología de la extremidad torácica en eventos agudos y crónicos.

Se concluye que la fuerza de prensión y la pinza 1 en manos dominantes y no dominantes están relacionadas con el peso, talla y el porcentaje de la grasa corporal y circunferencia de la muñeca, y ésta a su vez con el predominio en el sexo masculino que en el femenino, en personas económicamente activas.

Referencias

1. Coronel OMG, Hernández AH, Hernández JI. Determinación de la fuerza isométrica de prensión manual gruesa en población en edad laboral con dinamometría obtenida con el equipo terapéutico Baltimore. Rev Mex Med Fis Rehab. 2018; 20 (1-2): 5-11.

2. Martins JC, Aguiar LT, Lara EM, Texeira-Salmela LF, Faria CDCM. Assessment of grip strength with modified sphygmomanometer test: association between upper limb global strength and motor function. Braz J Phys Ther. 2015; 19 (6): 498-506. Available in: http//dx.doi. org/10.1590/biptrbf.2014.0118.

3. Kalyani RR, Kim C, Ferrucci L, Laughlin GA, Kritz-Silverstein $\mathrm{D}$, Kong S, et al. Sex differences in the associations of fasting and postchallenge glucose levels with grip strength among older adults: the rancho Bernardo Study. BMJ Open Diabetes Research and Care. 2015; 3: e00086. doi: 10.1136/bmjdrc-2015000086.

4. Guede F. Fuerza prensil de mano y su asociación con la edad, género y dominancia de extremidad superior en adultos mayores autovalentes insertos en la comunidad. In estudio exploratorio. Rev Med Chile. 2015; 146: 995-1000.

5. Yamada E, Takeuchi M, Kurata M, Tsuboi A, Kazumi T, Fukuo K. Low heamoglobin levels contribute to low grip strength independent of low-grade inflammation in Japanese elderly women. Asia Pac J Clin Nutri. 2015; 24 (3): 445-51.

6. Lam NW, Goh HT, Kamaruzzaman SB, Chin AV, Poi PJ, Tan MP. Normative data for hand grip strength and key pinch strength, stratified by age and gender for a multiethnic Asian population. Singapore Med J. 2015; 1-21.

7. Cuesta-Vargas A, Hilgenkamp T. Reference values of grip strength measured with a jamar dynamomenterin 1,526 adults with intelectual disabilites and compared to adults without intellectual disability. PloS One. 2015; 10 (06): e0129585. doi: 10.1371/journal. pone.0129585.

8. Hepping AM, Ploegmaker JJW, Geertzen JHB, Bulstra SK. The influence of hand preference on grip strenght in children and adolescents. PloSONE. 2015; 10 (11= e0143476. doi: 10.1371/journal. pone.0143476.

9. Pérez GA, Jurado TM, Sancho BJL. Fuerza de contacto entre mano y objeto en el agarre cilíndrico: comparación de dos técnicas de medición. Dpto. de Ingeniería Mecánica y construcción. Universitat Jaume I. XIX Congreso Nacional de Ingeniería mecánico.

10. Jean-Yves Hogrel. Grip Strength measured by high precisión dynamometry in healthy subjects from 5 to 80 years. Hogrel BMC Musculoskeletal Disorders. 2015; 16: 139.

11. Escalona D’AP, Naranjo OJ, Lagos SV, Solís FF. Parámetros de normalidad de fuerza de prensión de mano en sujetos de ambos sexo de 7 a 17 años de edad. Rev Chil Pediatr. 2009; 80 (5): 435-43.

12. Schreuders TA, Roebroeck ME, Goumans J, Van Nieuwenhuijzen JF, Stijnen TH, Stam HJ. Measurement error in grip and pinch force measurements in patient with hand injuries. Phys Ther. 2003; 83 (9): 806-15.

13. Peters MJ, Van Nes SI, Vanhoutte EK, Bakkers M, Van Doorn PA, Merkies IS, et al. Revised normative valvues for grips strength with the Jamar dynamometer. J Peripher Ner Syst. 2011; 16 (1): 47-50.

14. Angst F, Drerup S, Werle S, Harren DB, Summen BR, Goldhahn J. Predictions of grip and key pinch strength in 978 healthy subjects. BMC Musculoskelet Disord. 2010; 11: 94.

15. Lenardt MH, Hammerschmidt KC, Elero BS, Binotto MA, Damarys KM. Fatores Associados á forca de preensao manual dismuida em idosos. Esc Anna Nery. 2016; 20 (4): e20160082. 